\title{
Modifications performed to the Fermenter R1 at the Molecular Immunology Center
}

\author{
Waldo Hernández Sánchez ${ }^{1}$, Albo D. Domínguez ${ }^{2}$, Yusmeily Díaz Puebla ${ }^{3}, J^{\prime m m y ~ N u n ̃ e z ~}{ }^{4}$ \\ ${ }^{1,2,3,4}$ Centro de Inmunología Molecular - Calle 216 esq 15 Siboney Playa La Habana, Cuba.
}

Email: waldo@cim.sld.cu

Received: November $24^{\text {th }}, 2017$.

\begin{abstract}
For high demand in the global market for erythropoietin (EPO) and the inability to grow in new fermentation's devices, by high costs and services, it is necessary to provide to Molecular Immunology Center (CIM) fermentation's systems more efficient and that is the objective of our work collective. Among the main problems in this fermenter were: difficulties with some sequences of operation; high risks of operation by the operator as some sequences of operations are performed manually; failures (for automatic operation) in fermentation runs accounted for $65 \%$ of total failure of this equipment. This work is related to a series of changes in "software and design" made to the fermenter R1 of the CIM EPOVAC plant, which has reflected a progressive increase in productivity of the fermenter, reaching record production figures.

Accepted: November $25^{\text {th }}, 2017$.

Published: December $13^{\text {th }}, 2017$.

\section{Modificaciones realizadas al fermentador R1 del Centro de Inmunología Molecular}

\begin{abstract}
RESUMEN
Por la alta demanda existente en el mercado mundial de eritropoyetina (EPO) y la imposibilidad de crecer en nuevos dispositivos de fermentación por sus elevados costos y servicios, es necesario dotar al Centro de Inmunología Molecular (CIM) de sistemas de fermentación más eficientes y es ese el objetivo de nuestro colectivo de trabajo. Entre los principales problemas em este fermentador se encontraban: dificultades con algunas secuencias de funcionamiento; elevados riesgos de operación por parte de los operarios ya que algunas de las secuencias de operaciones se realizaban de forma manual; los fallos (por operación automática) en las corridas de fermentación representaban el $65 \%$ de las fallas totales de este equipo. Este trabajo está relacionado con una serie câmbios de "diseño y software" realizados al fermentador de R1 de la planta EPOVAC del CIM, lo que ha reflejado um aumento progresivo de la productividad de dicho fermentador, alcanzando cifras record de producción.
\end{abstract}

Palabras Claves: Fermentador, PLC, Software.

\section{INTRODUCCIÓN}

La biotecnología se puede definir como la utilización de organismos vivos, o partes de los mismos, para obtener o modificar productos, mejorar plantas o animales o desarrollar microorganismos para objetivos específicos. Así, se unen los conceptos tradicionales y los más modernos de la ingeniería genética configurándose como una ciencia multidisciplinaria que engloba entre otros la genética molecular, la ingeniería química y de proceso, la anatomía animal y vegetal, la bioquímica, la microbiología, la inmunología, la biologia celular, la agricultura y la electrónica [1].

Cuba se ha dedicado especialmente al desarrollo de la biotecnología en las últimas décadas, contando ya con varias instituciones que se dedican a la ingeniería genética, la investigación y a la producción de biofármacos, aportando así cuantiosos ingresos al país cada año, debido a las exportaciones y a la creación de empresas filiales en el extranjero. Gran parte del desarrollo biotecnológico que existe en la actualidad se basa en procesos de fermentación de tipo industrial, el Centro de Inmunología Molecular es uma institución que se desempeña en 
esta área y se dedica al estudio del cáncer y otras enfermedades, y a la producción de vacunas y medicamentos para el tratamiento de estos padecimientos.

Los biofármacos comercializados por esta empresa se basan en la fermentación continua de células animales y se producen en las plantas de fermentación del propio centro. El buen estado de estas plantas es un requisito clave para poder alcanzar los niveles de calidad requeridos en la obtención de estos biofármacos, debido a esto resulta de vital importância lograr que estos procesos tengan un nivel alto de automatización, con sistemas capaces de controlar los distintos procesos de forma eficiente y de mantener las variables fundamentales en un fermentador, dentro de los parâmetros establecidos.

En estos procesos se controlan variables como temperatura, $\mathrm{pH}$, presión, $\mathrm{O}_{2}$ disuelto, nivel en el interior del fermentador y velocidad de agitación. Estas variables deben mantenerse dentro de un intervalo de valores específicos, garantizando así las condiciones necesarias para que el cultivo celular crezca y se desarrolle adecuadamente. Lograr esto no resulta una tarea sencilla, se necesita de forma general de sensores con buenas características para la medición de cada variable, de transmisores y de un autómata programable por ser estos, procesos de alta complejidad que trabajan con un elevado número de variables. Además se requiere un sistema de supervisión que permita conocer el estado del proceso en cada momento, almacenar información acerca del comportamento del mismo y emitir alarmas en caso de que se produzcan situaciones anormales.

\section{I.1 SITUACIÓN PROBLEMA}

Se detectaron una serie de problemas automáticos que impedían el aumento y la calidad de la producción del fermentador:

- Dificultades con algunas secuencias de funcionamiento del fermentador, lo que desencadenó en contaminaciones del médio de cultivo y bajos niveles de productividad.

- Elevados riesgos de operación ya que algunas de las secuencias de operaciones se realizaban de forma manual, repercutiendo en la calidad del producto.

- Los fallos (por operación automática) en las corridas de fermentación representaban el $65 \%$ de las fallas totales en el año 2013.

- Necesidad de instalación de nuevos elementos de control, con el objetivo de facilitar la operación, aumentar el nivel de confiabilidad del equipo y la productividad.

- Repetidas asistencias técnicas por parte de los fabricantes del equipo para solucionar los problemas que iban surgiendo. (KNOW HOW del fabricante).

\section{I.2 OBJETIVO GENERAL}

Realizar modificaciones al diseño y software al fermentador R1 de la planta de EPOVAC del CIM para garantizar um aumento de la productividad y la seguridad del sistema.

\section{ASPECTOS GENERALES DE LOS PROCESOS DE FERMENTACIÓN}

Un proceso de fermentación típico es esencialmente um proceso que se lleva a cabo en un recipiente llamado fermentador o biorreactor, mediante el cual determinados sustratos que componen el medio de cultivo son transformados por acción microbiana en metabolitos y biomasa. El microorganismo va aumentando en su concentración en el transcurso del proceso al mismo tiempo que el medio se va modificando y se forman productos nuevos como consecuencia de las atividades catabólicas y anabólicas. Los dos fenómenos crecimiento y formación de producto, tienen lugar durante el desarrollo del proceso simultáneamente o no, según los casos [2].

Resumiendo, un proceso fermentativo consiste en unir un medio de cultivo que contiene nutrientes con un microorganismo dado, en un fermentador, donde se establecen condiciones experimentales adecuadas para obtener finalmente el producto deseado.

\section{II.1DESCRIPCIÓN TECNOLÓGICA DEL PROCESO DE FERMENTACIÓN DEL FERMENTADOR R1 DE LA PLANTA EPOVAC.}

La planta EPOVAC del CIM, se dedica a la producción de la eritropoyetina, el cultivo de las células se realiza mediante el uso de un fermentador de tanque agitado que opera en modo perfusión, como se muestra en la figura 1 .

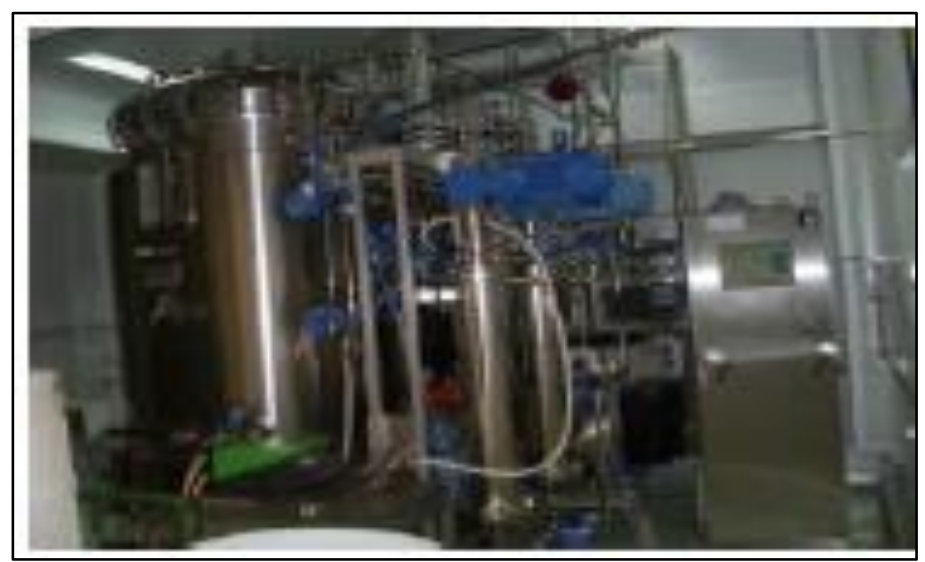

Figura 1: Fermentador R1.

Fuente: Autores, (2017).

Este fermentador es del fabricante BIOENGINEERING de acero inoxidable con $1500 \mathrm{~L}$ de capacidad neta, de los cuales se usan $1000 \mathrm{~L}$ para el proceso productivo [3].

\section{II.1.1 VARIABLES CONTROLADAS EN EL PROCESO}

- Nivel;

- Presión;

- $\mathrm{pH}$;

- Oxígeno disuelto;

- Temperatura en el vaso;

- Temperatura (puntos fríos);

- Conductividad;

- Velocidad de agitación (vaso);

- Flujo de gases;

- Velocidad de agitacion (Rotofilter);

- Flujo de cosecha.

En el fermentador se realizan 3 tipos de atividades automatizadas fundamentales que garantizan su correcto funcionamiento:

\section{- CIP (cleaning in place)}

Antes de comenzar el proceso de fermentación es necessário que el biorreactor se encuentre limpio, con este 
objetivo se realiza el proceso de limpieza en el lugar (cleaning in place).

Este proceso se lleva a cabo con la ayuda de una bomba de recirculación que se encarga de distribuir tanto a las líneas como al vaso del fermentador las soluciones de limpieza y agua purificada para las fases de enjuague. Una vez finalizados los enjuagues se realiza un arrastre con aire a presión para dejar seco el fermentador y así queda listo para iniciar la esterilización. Toda la operación de limpeza funciona de forma automática.

\section{- SIP (Sterilization in place)}

La esterilización comienza por los filtros de entrada y salida de gases, empleando para ello vapor limpio, com temperaturas mayores a $121^{\circ} \mathrm{C}$, durante unos 30 minutos. Luego se procede a la esterilización de la línea de adición de medio, la conexión de las bolsas de adición de medio al fermentador y la línea de cosecha respectivamente, manteniendo las condiciones de esterilización mencionadas anteriormente. Toda la operación de esterilización funciona de forma automática.

\section{- Fermentación}

Cuando el sistema ya ha sido enfriado se realiza la transferencia del cultivo hasta el puerto de inóculo del fermentador de producción. A las 24 horas de iniciado el processo fermentativo y aproximadamente cada 48 horas, se toman muestras para seguir el comportamiento de la concentración y de la viabilidad celular, control del pH, contaminación microbiana, concentración del producto y concentración de glucosa.

La extracción del sobrenadante se realiza mediante un par de filtros rotatorios externos (Rotofilter), y mediante un sistema de tuberías, la biomasa residual de los filtros se devuelve al medio de fermentación. Este proceso termina cuando las células hayan alcanzado su máximo rendimiento, lo cual abarca normalmente un período de tres meses.

\section{SISTEMA DE CONTROL DEL FERMENTADOR}

El sistema control del fermentador está basado en periferia descentralizada utilizando un bus de comunicación (Profibus) para enlazar los módulos de periferia. Estos módulos son la interfaz entre el Controlador Lógico Programable (PLC) y los dispositivos de campo (sensores y actuadores) A continuación se muestra la estructura del sistema de control del fermentador (Véase Figura 2).

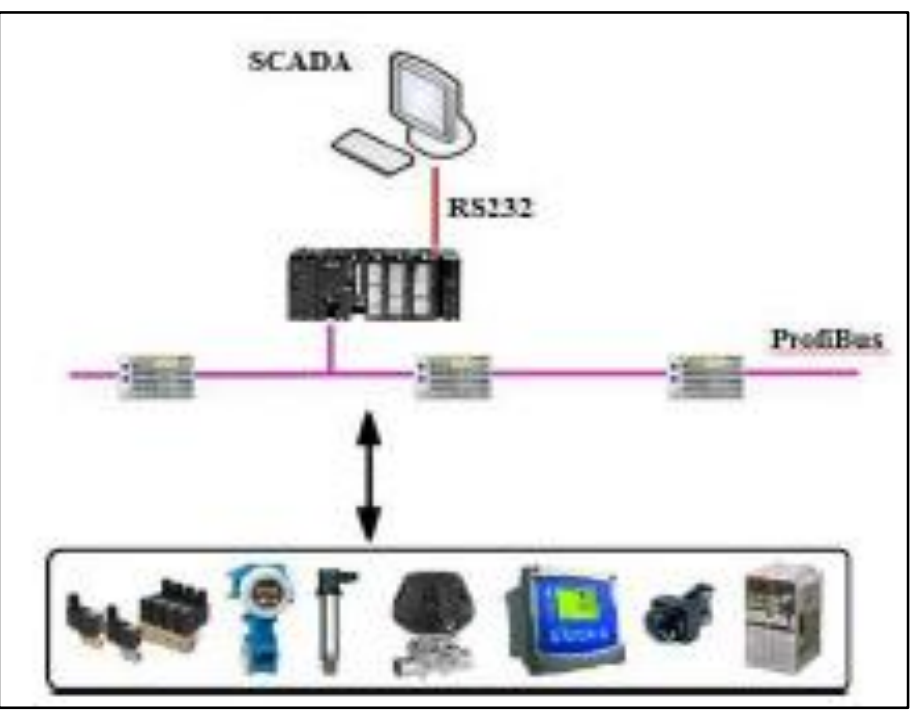

Figura 2: Sistema de control del fermentador.

Fuente: Autores, (2017).

\section{III.1 CONTROLADOR LÓGICO PROGRAMABLE (PLC)}

Un Autómata Programable Industrial (API) o Controlador Lógico Programable (PLC), es una máquina electrónica programable diseñada para ser utilizada en un entorno industrial (hostil), que utiliza una memoria programable para el almacenamiento interno de instrucciones orientadas al usuario, para implantar soluciones específicas tales como funciones lógicas, secuencias, temporizaciones, recuentos y funciones aritméticas, con el fin de controlar mediante entradas y salidas, digitales y analógicas diversos tipos de máquinas o procesos. [4]

Las señales de entrada pueden proceder de elementos digitales, como finales de carrera, detectores de proximidad o presencia, o analógicos, como sensores de temperatura y dispositivos cuya salida sea tensión o corriente continua. Referente a las señales de salida existen dos tipos; las salidas digitales que pueden ser analizadas como 1 ó 0 (todo o nada) y las salidas analógicas en tensión o corriente, estas salidas se envían a elementos indicadores y actuadores del proceso, como pueden ser lámparas, contactores, válvulas, entre otros.

El autómata gobierna las señales de salidas según la lógica del programa de control previamente almacenado en una memoria en su unidad de procesamiento central $(C P U)$, a partir del estado de las señales de entrada. Este programa se inserta en el PLC a través de la unidad de programación, que permite además funciones adicionales como depuración de programas, simulación, monitorización, control del autómata y otras más.

La CPU encargada de realizar el control de todas las operaciones del fermentador y garantizar su correcto funcionamiento y seguridad es un S7-300 del fabricante SIMENS. En este se realizan la mayor parte de las modificaciones para garantizar un mejor funcionamiento del sistema de control. El sistema de control del fermentador se encuentra programado en dos lenguajes de programación, el KOP y GRAPH 7.

\section{III.1.1 KOP}

La representación del lenguaje de programación gráfico KOP (esquema de contactos) es similar a la de los esquemas de circuitos. Los elementos de un esquema de circuitos, tales como los contactos normalmente cerrados y normalmente abiertos, se agrupan en segmentos. Uno o varios segmentos constituyen el área de instrucciones de un bloque lógico [5].

\section{III.1.2 GRAPH7}

El lenguaje gráfico de programación S7-Graph permite programar controles secuenciales. Comprende la creación de uma cadena de etapas, la definición de los contenidos de las mismas y las condiciones de transición. El contenido de las etapas se define con un lenguaje de programación especial (similar a AWL), em tanto que las condiciones de transición se introducen en uma representación del esquema de contatos (parte del lenguaje de programación KOP). S7-Graph permite representar también procesos complejos de forma muy clara, permitiendo así una programación y una búsqueda de errores efectivas [6].

\section{MODIFICACIONES Y CAMBIOS AL SISTEMA DE CONTROL}

Se realizaron un total de 18 modificaciones a las secuencias de programa y parámetros de operación del fermentador, así como se incorporaron cambios al diseño original del equipo con el objetivo de alcanzar mejores prestaciones en su funcionamiento y aumentar la calidad y rendimiento del producto. 
A continuación se muestra una imagen del software STEP7 y el programa de control del fermentador (Véase figura 3), en el cual se realizaron las modificaciones y se actualizaron en el
PLC mediante una laptop, realizando un enlace Multi Point Interface (MPI) con la CPU.

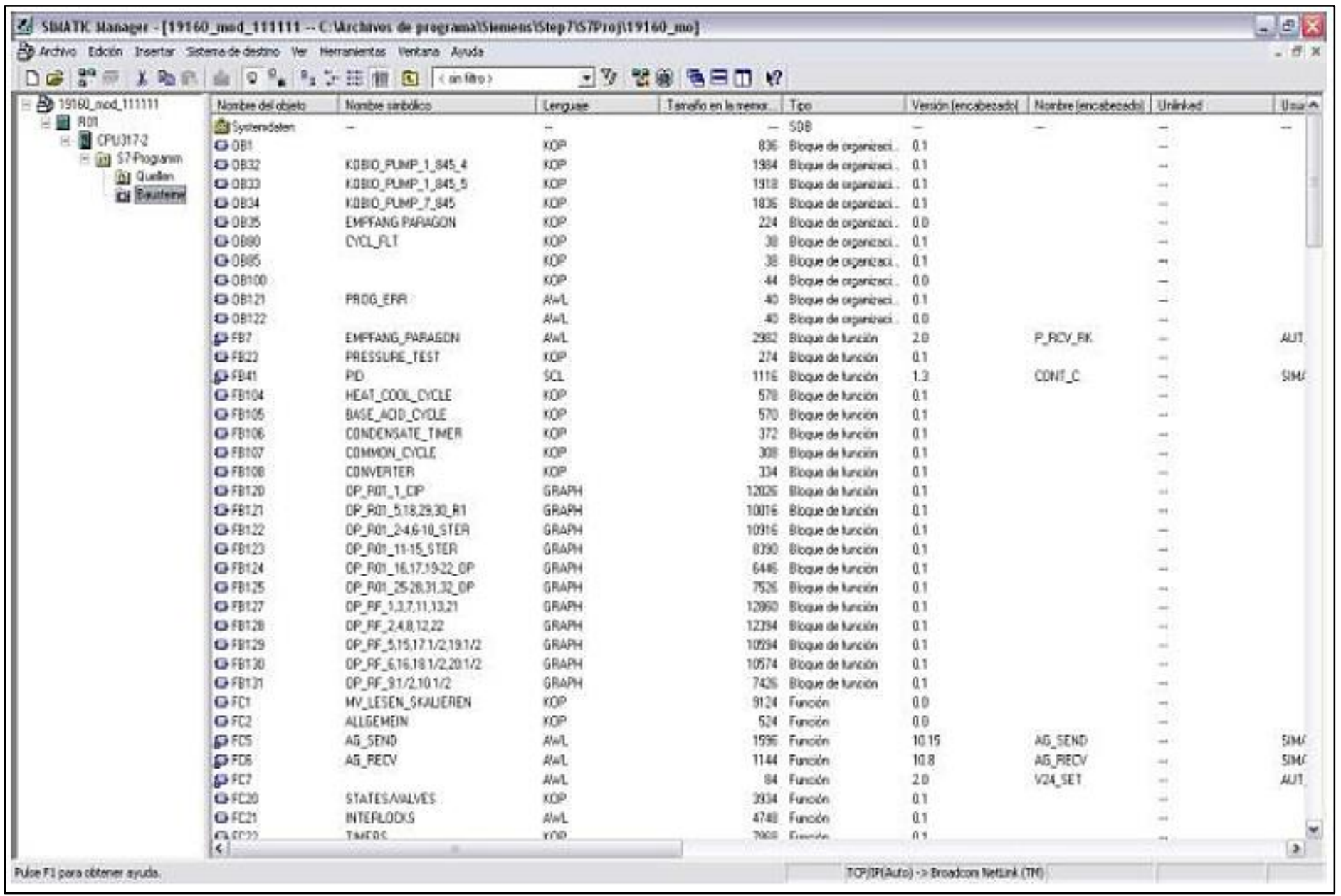

Figura 3: Programa del fermentador en STEP7.

Fuente: Autores, (2017).

\section{IV.1 PRINCIPALES MODIFICACIONES REALIZADAS}

Se modificaron varias secuencias de programa en las cuales existían aperturas de válvulas no deseadas em varias secuencias de operación, lo que presentaba um alto riesgo de contaminación en el fermentador. A continuación se muestra el SCADA, acrónimo de (Supervisory Control And Data Adquisition) con las válvulas re-configuradas (Véase figura 4).

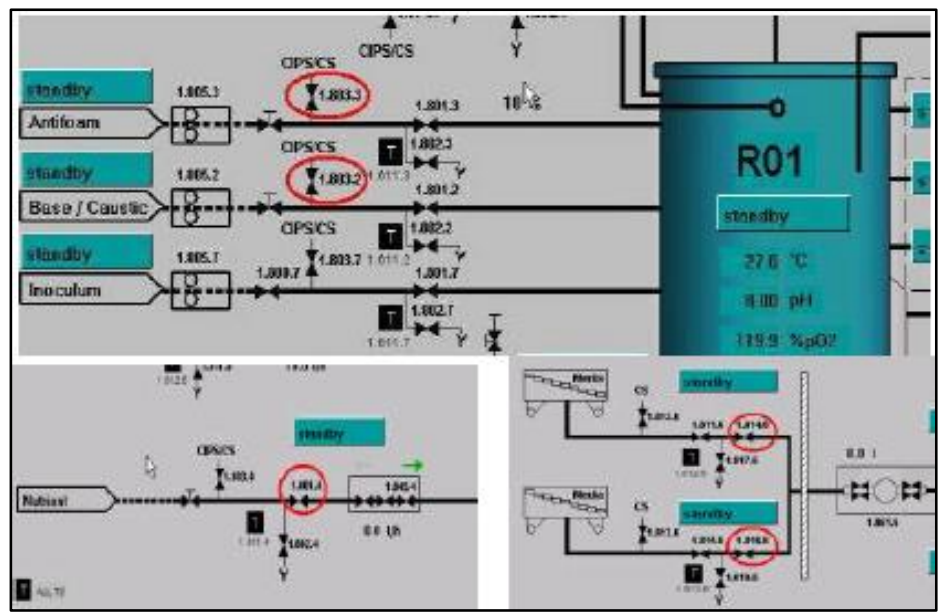

Figura 4: Modificaciones a secuencias de válvulas.

Fuente: Autores, (2017).
Se adicionaron 2 válvulas de control con sus respectivas secuencias de operación con el objetivo de garantizar una correcta esterilización del sistema (Véase figura 5).

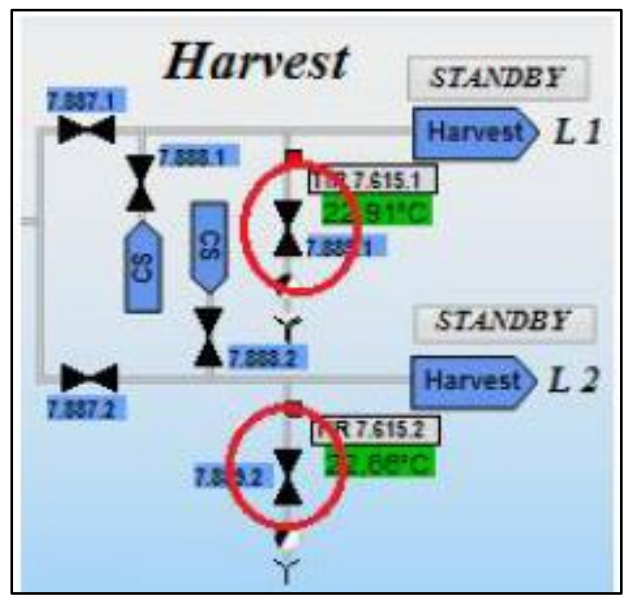

Figura 5: Válvulas de control incorporadas.

Fuente: Autores, (2017).

Se realizó una modificación en el funcionamiento de lab bomba de recirculación de la chaqueta del fermentador durante la esterilización del sistema, para evitar cavitaciones en su interior (Véase figura 6). 


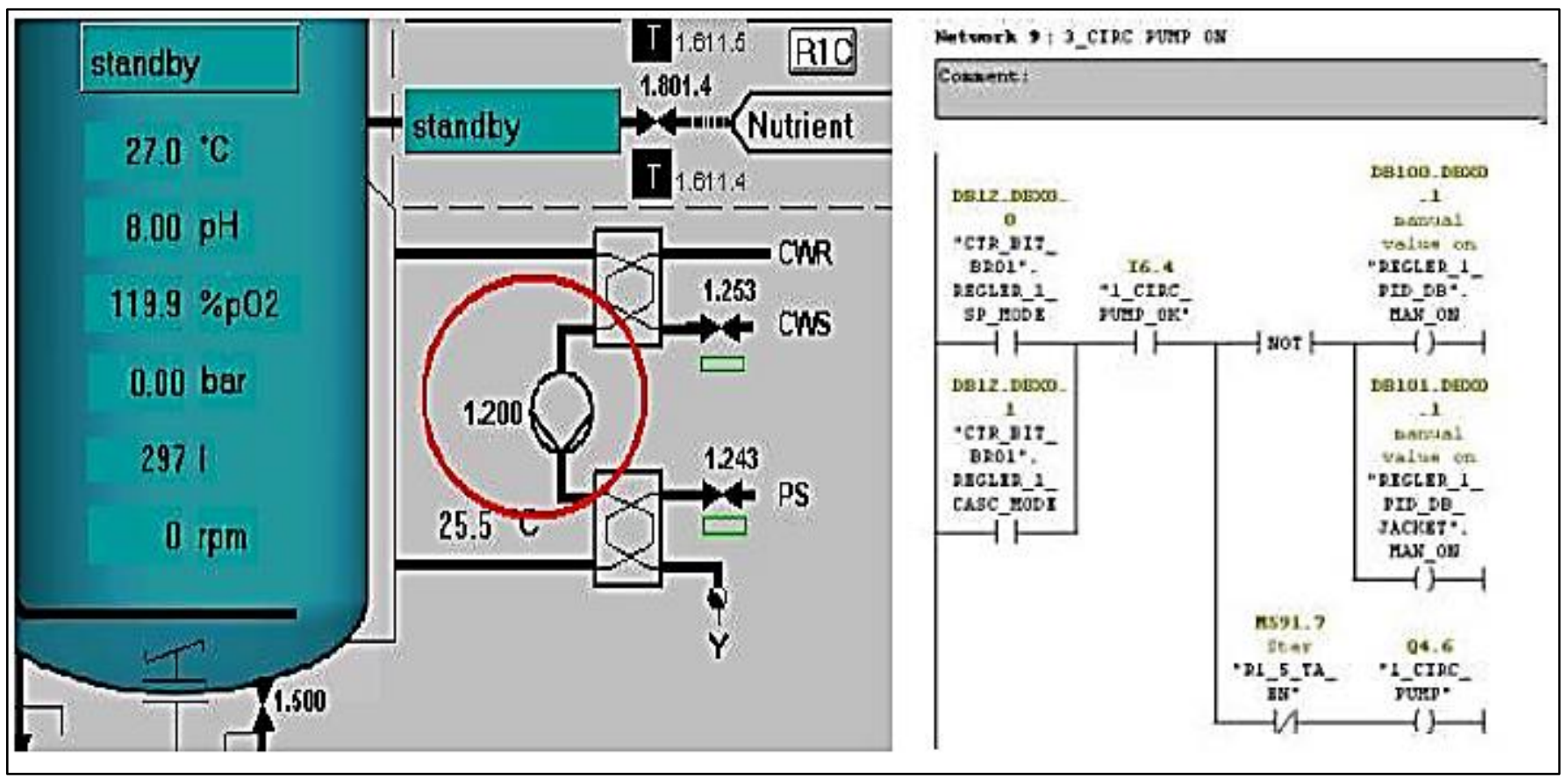

Figura 6: Modificación a la secuencia de la operación de la bomba.

Fuente: Autores, (2017).

Se realizó la incorporación de un nuevo sensor de flujo al sistema, lo que brinda la posibilidad de visualizar el flujo de cosecha y controlar dicha variable. (Véase figura 7).

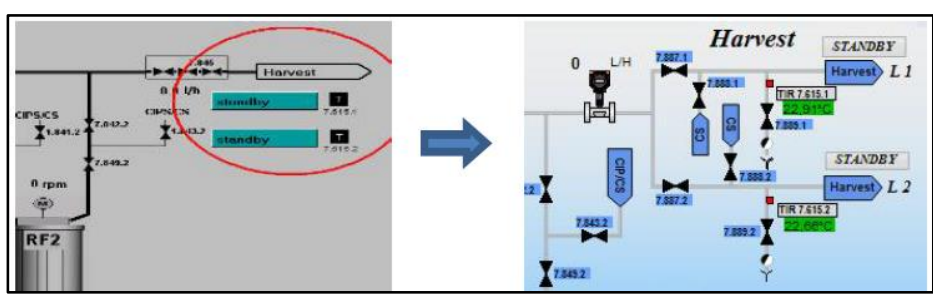

Figura 7: Incorporación de sensor de flujo en la línea de cosecha. Fuente: Autores, (2017).

Se realizó el correcto ajuste y linealización de las RPM de los motores de los RotoFilter, modificando las secuencias correspondientes con el objetivo de aumentar la velocidad de los mismos, de $500 \mathrm{rpm}$ a $800 \mathrm{rpm}$. (Véase figura 8.) Esta modificación se realiza con el objetivo de alcanzar un mayor grado de retención celular en el interior del Rotofilter, así como minimizar la tupición de los filtros.

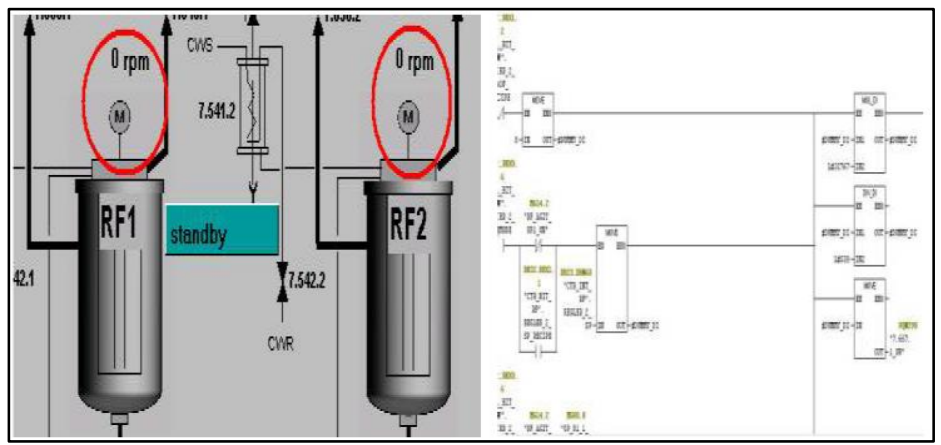

Figura 8: Ajuste de lazo de control de velocidade.

Fuente: Autores, (2017).

Se realizó la modificación de una secuencia de programa que no permitía realizar la operación de INOCULO (operación deincorporar las células al interior del fermentador) de forma automática (Véase figura 9.).

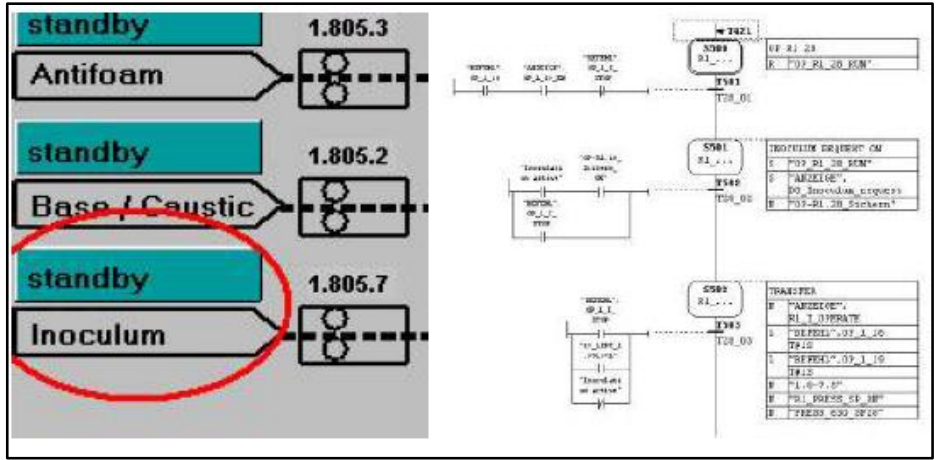

Figura 9: Modificación de secuencia de operación de INOCULO. Fuente: Autores, (2017).

Se realizó la modificación del sistema de CIP en los RotoFilter, donde se agregaron válvulas, sensores y se modificaron y adicionaron nuevas secuencias de programa relacionadas con el CIP de los RotoFilter. Lo que garantiza una limpieza superior de los filtros de los Rotofilter en comparación con la forma en se realizaba por diseño original del sistema (Véase figura 10).

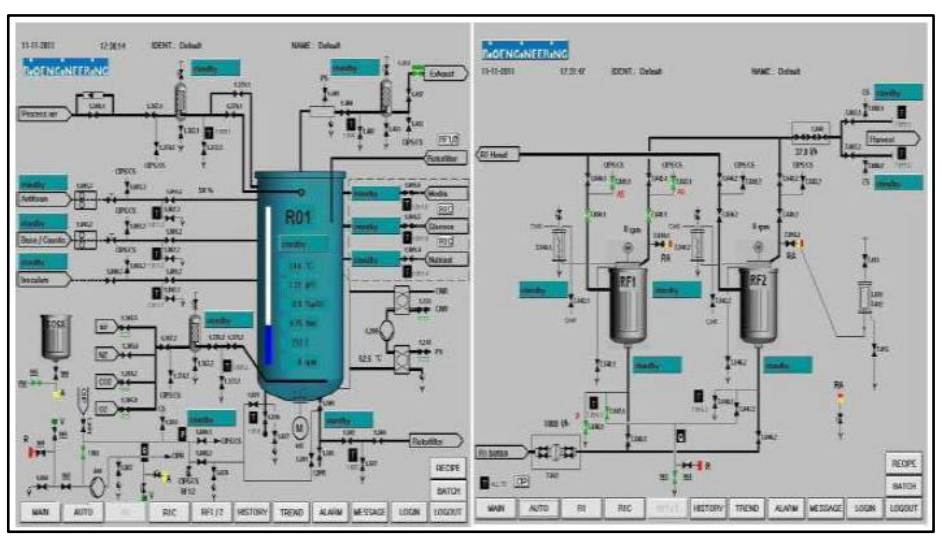

Figura 10: Modificaciones al CIP de los RotoFilter.

Fuente: Autores, (2017). 
También se realizaron modificaciones de software referentes a:

- Los status informativos en el SCADA de las operaciones.

- Calibración de las bombas DOMP PUMP de adición de medio de cultivo y recirculación de sobrenadante.

- Tiempos de pulsación de las válvulas durante la esterilización.

\section{RESULTADOS Y DISCUSIÓN}

Entre los resultados alcanzados más relevantes se encuentran:

1. Con el aumento de las RPM y las modificaciones realizadas al CIP, se reflejó un aumento sustancial del rendimiento de los RotoFilters funcionamiento continuo, eliminando interrupciones en la cosecha y manipulaciones innecesarias para realizar el CIP. A continuación (Véase Figura 11) se muestran dos gráficas comparativas que muestran el desempeño de los RotoFilters en 60 días de uso.

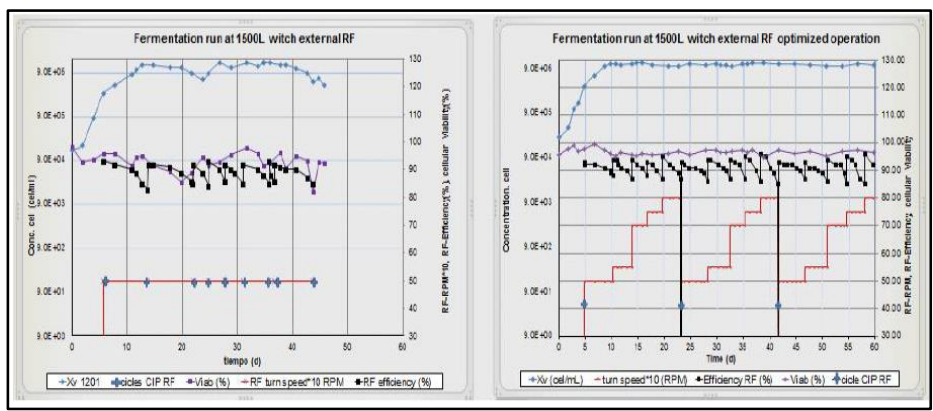

Figura 11: Desempeño de los RotoFilters.

Fuente: Autores, (2017).

2. Con la implementación de estas modificaciones y unido a nuevas técnicas de fermentación implementadas por parte de los tecnólogos de la Planta ha reflejado un aumento progresivo de la productividad de dicho fermentador, alcanzando cifras record de producción. El incremento de la concentración celular de 6-8 millones cell/ $/ \mathrm{mL}$ (año 2010) a 10-13 millones cell/mL (año 2014) aumentando la masa neta de EPO obtenida en casi 2,5 veces por mes, lo que representa un aumento de aproximadamente 3 millones de dólares exportados en cada mes. En las siguientes figuras se muestran las gráficas comparativas de las principales variables productivas en ambos años.

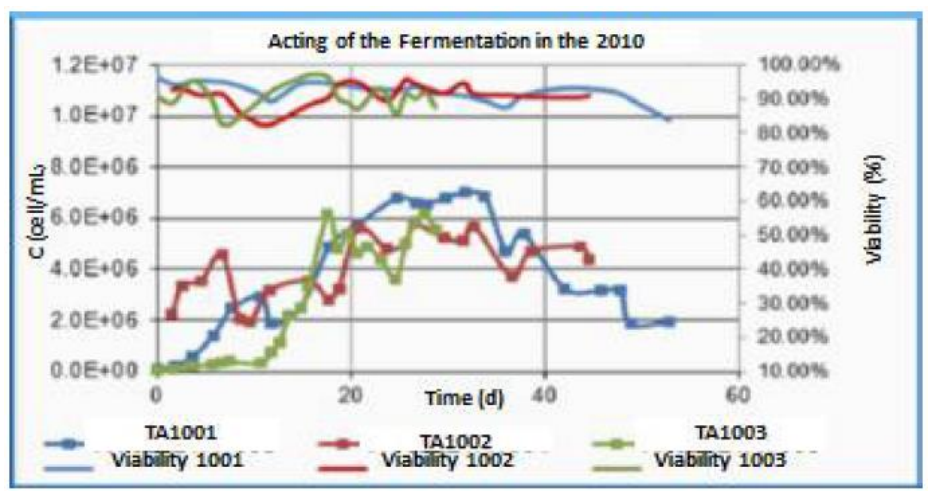

Figura 12: Concentración celular año 2010.

Fuente: Autores, (2017).

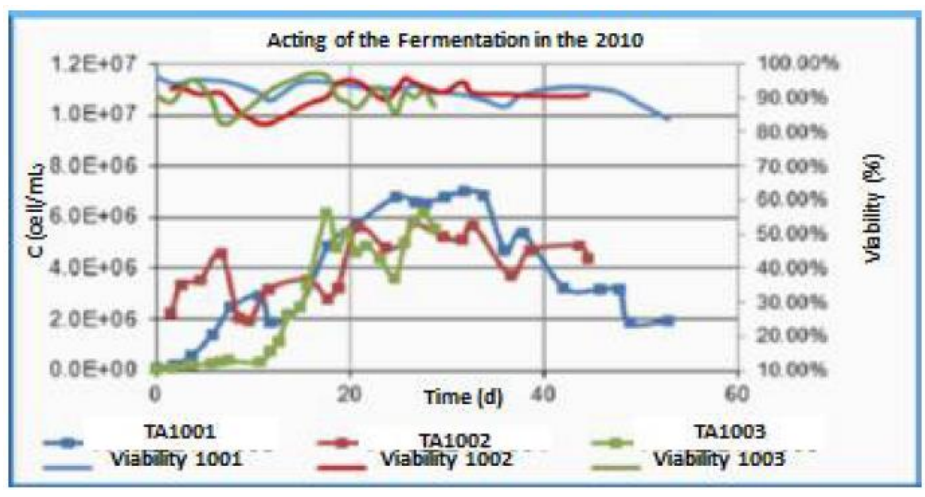

Figura 13: Concentración celular año 2014.

Fuente: Autores, (2017).

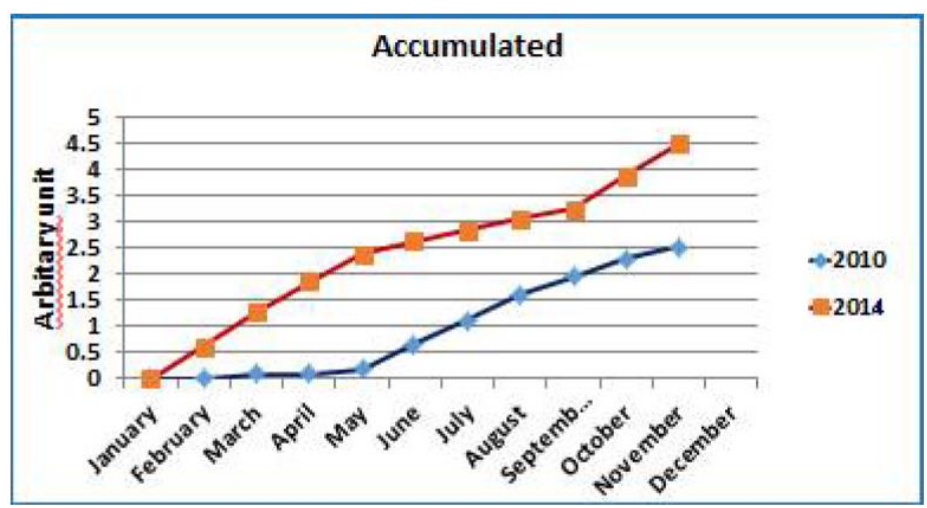

Figura 14: Acumulado de producto final.

Fuente: Autores, (2017).

3. El impacto de contaminaciones por problemas automáticos se ha "eliminado" durante el año 2014, así como se han minimizado considerablemente el número de fallas (por operaciones automáticas) en el fermentador, de 21 fallas reportadas en el año 2013 se redujo a 4 fallas no críticas en 2014.

4. Se realizó una validación del sistema de control de acuerdo a las modificaciones realizadas, obteniendo resultados satisfactorios para el correcto desempeño del fermentador.

\section{CONCLUSIONES}

1. Con estas modificaciones se contribuyó a elevar la productividad del centro, a niveles jamás alcanzados.

2. Se adaptó el sistema de control del fermentador a las necesidades propias de la Planta de Producción.

3. Se elevó el nivel de confiabilidad, funcionamiento y operación, así como el nivel de seguridad en las operaciones.

4. Se logra adaptar y convertir el Know How del fabricante en un Know How propio del CIM.

\section{BIBLIOGRAFÍA GENERAL}

[1]. Biología Moderna [Internet], mayo 2012. Disponible en: http://biologiamodernaa.blogspot.com/2012/05/acidosnucleicos. html.

[2]. Cuellas A. Biorreactores Teoría, Universidad Nacional de Quilmes, Argentina 2007.

[3]. Nuñez J. Filtración de sobrenadante de eritropoyetina producida en tanque agitado. Centro de Inmunología Molecular; 2010. 
[4]. Alvarado F.E. Guía de Prácticas para autómata programable basado en el S7-200 y el EM-235, Universidad de ANZUAY, Ecuador, 2010.

[5]. Capítulo 4, Controlador Lógico Programable PLC [Internet], Disponible en: http://catarina.udlap.mx/u_dl_a/tales/documentos/lmt/maza_c_acc apitulo4.pdf.

[6]. Manual Programar con STEP7 [Internet], 2006, Disponible en:http://cache.automation.siemens.com/dnl/jExNDU0NwAA_16 52056_HB/S7prv54_s.pdf.

[7]. Dellamea AB. Eritropoyetina humana recombinante [Internet]. Centro deDivulgación Científica, Facultad de Farmacia ny Bioquímica, Universidad de Buenos Aires.; 2013. Disponible en: http://www.edicionesmedicas.com.ar/.

[8]. Siemens. Central Processing Unit CPU 314. [Internet]. 2007. Disponible en: www.siemens.com.

[9]. Siemens. Configurar el hardware y la comunicación con Step 7. [Internet]. 2006. Disponible en: www.siemens.com.

[10]. Siemens. Esquema de contactos (KOP) para S7-300 y S7400. [Internet]. 2006. Disponible en: www.siemens.com.

[11]. Pregi N. Estudio de la eritropoyetina como agente neuroprotector. [Tesis Doctoral]. [Buenos Aires, Argentina]: Universidad de Buenos Aires; 2008.

[12]. Bouzó L, Arias MA, Suárez J, Ojito E, Rodríguez E, Alvarez A, et al. Optimización de la fermentación en fermentadores de fibra hueca para la producción de biofármacos. Centro de Inmunología Molecular, La Habana, Cuba; 2002.

[13]. Cruz J, Villalta D, Curbelo D, García Z, Vallín A, Portillo $\mathrm{A}$, et al. Preparaciones de eritropoyetina libres de albúmina. Centro de Inmunología Molecular, La Habana, Cuba; 2002.

[14]. Siemens. S7-300 CPU 31xC and CPU 31x: Technical Specifications. [Internet]. 2011. Disponible en: www.siemens.com. 